\title{
Lipoxygenase Inhibitory Effects of Dibenzylbutane Lignans from the Seeds of Myristica fragrans (Nutmeg)
}

\author{
Hyun Sook Kwon, Soo Jeong Cho, ${ }^{\dagger}$ Tae Joung Ha, ${ }^{\ddagger}$ Amaravadhi Harikishore,${ }^{\S}$ Ho Sup Yoon, ${ }^{\S}$ \\ Ki Hun Park, "Il Suk Kim, and Dae Sik Jangף,"*
}

\author{
Department of Animal Resources Technology, Swine Science and Technology Center, and Department of Pharmaceutical \\ Engineering, Gyeongnam National University of Science and Technology, Jinju 660-758, Korea \\ ${ }^{+}$Department of Functional Crop, National Institute of Crop Science, RDA, Miryang 627-803, Korea \\ ${ }^{\S}$ Division of Chemical Biology \& BioTechnology, Nanyang Technological University, Singapore 637551

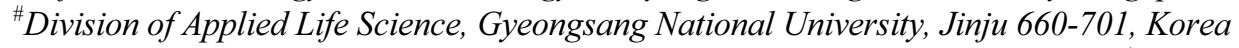 \\ 'Department of Life and Nanopharmaceutical Science, Kyung Hee University, Seoul 130-701, Korea. "E-mail:dsjang@khu.ac.kr \\ Received May 30, 2014, Accepted June 13, 2014
}

Key Words : Myristica fragrans, Nutmeg, Dibenzylbutane lignans, Soybean lipoxygenase, Molecular docking

Nutmeg (the seeds of Myristica fragrans Houtt) is a spice widely used in food as thin slices or more generally as a powder. Nutmeg is used in numerous recipes, including a lot of desserts (e.g. fruit cakes, muffins, pies) but in main courses as well (e.g. potato dishes, sauces). ${ }^{1}$ Nutmeg has been shown to possess various biological and pharmacological activities including anti-carcinogenic, ${ }^{2}$ anti-inflammatory, ${ }^{3}$ protein tyrosine phosphatase $1 \mathrm{~B}$ (PTP1B), ${ }^{4}$ and hepatoprotective activities. ${ }^{5}$ Previous phytochemical investigation on nutmeg have resulted in the isolation and identification of essential oils, ${ }^{6}$ lignans, ${ }^{7}$ and phenylpropanoids. ${ }^{8}$ Lipoxygenases (EC 1.13.11.12) are non-heme iron-containing enzymes that catalyze the site-specific oxygenation of polyunsaturated fatty acids to produce hydroperoxides. Lipoxygenases are suggested to be involved in the early event of atherosclerosis by inducing plasma low-density lipoprotein (LDL) oxidation. ${ }^{9,10}$ On the other hand, it is well known that lipid peroxidation is one of the major factors in deterioration during the storage and processing of foods, because it can lead to the development of unpleasant rancid or off flavors as well as potentially toxic end products. ${ }^{11}$ Hence, lipoxygenase inhibitors should have broad applications. ${ }^{12}$ Previously, we reported on the isolation of threoaustrobailignan-5 (1), macelignan (2), meso-dihydroguaiaretic acid (MDGA, 3), (-)-7-methyl ether dibenzylbutane lignan (4), myristargenol A (5), and isootobaphenol (6) from the seeds of $M$. fragrans and low-density lipoprotein (LDL)antioxidant activity of 1-6 and demethyldihydroguaiaretic acid (DDGA, 7). ${ }^{7}$ In our preliminary assay, the $\mathrm{MeOH}$ extract of seeds of $M$. fragrans completely inhibited the peroxidation of linoleic acid catalyzed by soybean lipoxygenase-1 (EC 1.13.11.12, Type 1) at $150 \mu \mathrm{g} / \mathrm{mL}$. In the present work, we present soybean lipoxygenase inhibitory activity of 1-7, the enzymatic inhibition kinetics, and molecular docking study.

We evaluated the abilities of 1-7 to inhibit soybean lipoxygenase- 1 and the effects of the compounds were assessed using $\mathrm{IC}_{50}$ values (Table 1).

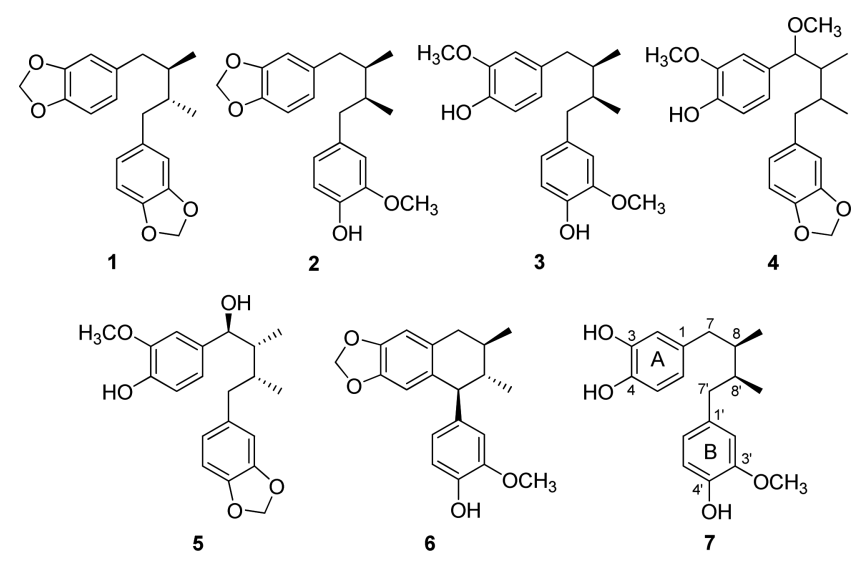

Figure 1. Chemical structures of the lignans 1-7.

Table 1. Inhibitory effects of 1-7 on soybean lipoxygenase-1 activity

\begin{tabular}{cc}
\hline Compounds & $\mathrm{IC}_{50}(\mu \mathrm{M})^{a}$ \\
\hline $\mathbf{1}$ & $>200$ \\
$\mathbf{2}$ & $168.90 \pm 0.9$ \\
$\mathbf{3}$ & $25.70 \pm 0.1$ \\
$\mathbf{4}$ & $195.00 \pm 0.5$ \\
$\mathbf{5}$ & $177.30 \pm 0.7$ \\
$\mathbf{6}$ & $180.00 \pm 1.0$ \\
$\mathbf{7}$ & $0.46 \pm 0.2$ \\
NDGA $^{\mathrm{b}}$ & $0.20 \pm 0.1$
\end{tabular}

${ }^{a}$ Compounds were examined in set of experiments repeated three times. ${ }^{b}$ NDGA (nordihydroguaiaretic acid) was used as a positive control.

As result, compounds $\mathbf{3}$ and $\mathbf{7}$ exhibited a strong inhibitory activity against soybean lipoxygenase-1. Macelignan (2), ( \pm ) 7-methyl ether dibenzylbutane lignan (4), myristargenol A (5) and isootobaphenol (6) showed little inhibitory activity, whereas threo-austrobailignan-5 (1) did not show any inhibitory effect on soybean lipoxygenase-1 up to $200 \mu \mathrm{M}$.

Our results showed that MDGA (3) and DDGA (7) inhibit catalytic activity of the lipoxygenase as a function of 

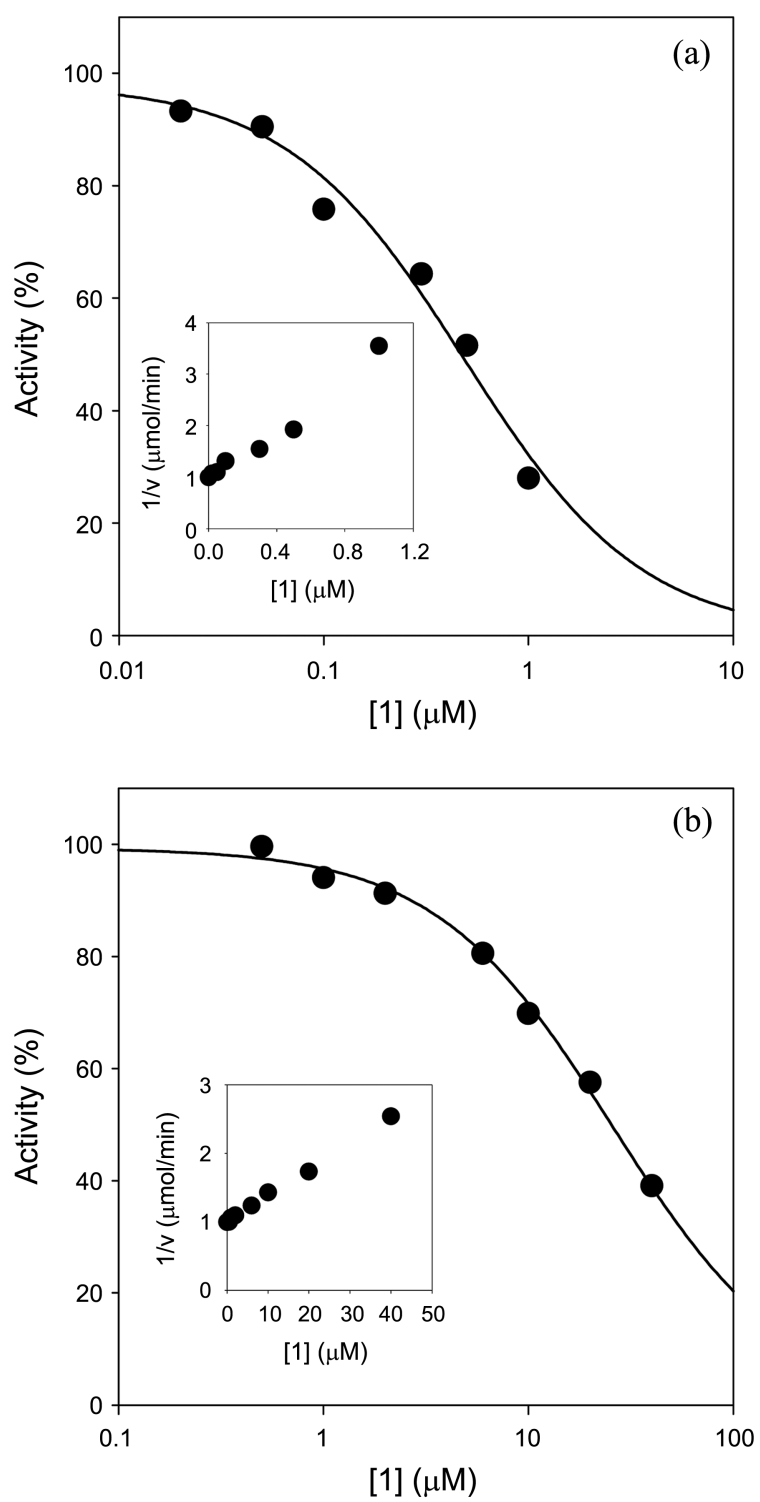

Figure 2. Effects of 7 (a) and 3 (b) on the activity of soybean lipoxygenase- 1 for the catalysis of linoleic acid at $25^{\circ} \mathrm{C}$. (Inset) Replots of data as $1 / v$ versus [I].

increasing concentrations of the compounds (Figure 2). The kinetic behavior of soybean lipoxygenase-1 oxidation of linoleic acid catalyzed by soybean lipoxygenase-1 follows Michaelis-Menten kinetics. The kinetic parameters for soybean lipoxygenase by DDGA (7) obtained from a Dixon plot showed that $K_{\mathrm{m}}$ is $18.6 \mu \mathrm{M}$ and $V_{\max }$ is $1.9 \mu \mathrm{M} / \mathrm{min}$ (Table 2). The estimated value of $K_{\mathrm{m}}$ obtained with a spectrophotometric method is in good agreement with the previously reported value. ${ }^{13,14}$

The kinetic and inhibition constants obtained are listed in Table 2. As illustrated in Figure 3, the inhibition kinetics analyzed by Dixon plots showed that DDGA (7) is a competitive inhibitor, since increasing DDGA (7) concentration resulted in a common intercept above the [I] axis but with different slopes. The equilibrium constant for inhibitor binding, $K_{i}$, was obtained from a plot of the apparent Michaelis-
Table 2. Kinetics and inhibition constants of soybean lipoxygenase-1 by MDGA (3) and DDGA (7)

\begin{tabular}{|c|c|c|}
\hline & \multicolumn{2}{|c|}{ Compounds } \\
\hline & 3 & 7 \\
\hline $\mathrm{IC}_{50}$ & $25.7 \mu \mathrm{M}$ & $0.46 \mu \mathrm{M}$ \\
\hline$K_{\mathrm{m}}$ & $17.1 \mu \mathrm{M}$ & $18.6 \mu \mathrm{M}$ \\
\hline$V_{\mathrm{m}}$ & $1.8 \mu \mathrm{M} / \mathrm{min}$ & $1.9 \mu \mathrm{M} / \mathrm{min}$ \\
\hline Inhibition & Reversible & Reversible \\
\hline Inhibition Type & Competitive & Competitive \\
\hline$K_{\mathrm{i}}$ & $13.6 \mu \mathrm{M}$ & $0.25 \mu \mathrm{M}$ \\
\hline
\end{tabular}

Menten constant versus the concentration of DDGA (7), which is linear. The inhibition kinetics analyzed by Lineweaver-Burk plots also confirmed that the DDGA (7) is a competitive inhibitor (data not illustrated). In addition, the inhibition kinetics analyzed by Dixon plots indicates that MDGA is also competitive inhibitor. The inhibition constant $\left(K_{i}=13.6 \mu \mathrm{M}\right)$ was obtained as shown in Table 2.

Thus, our studies confirmed that MDGA (3) and DDGA (7) are potent lipoxygenase inhibitors. Structure activity
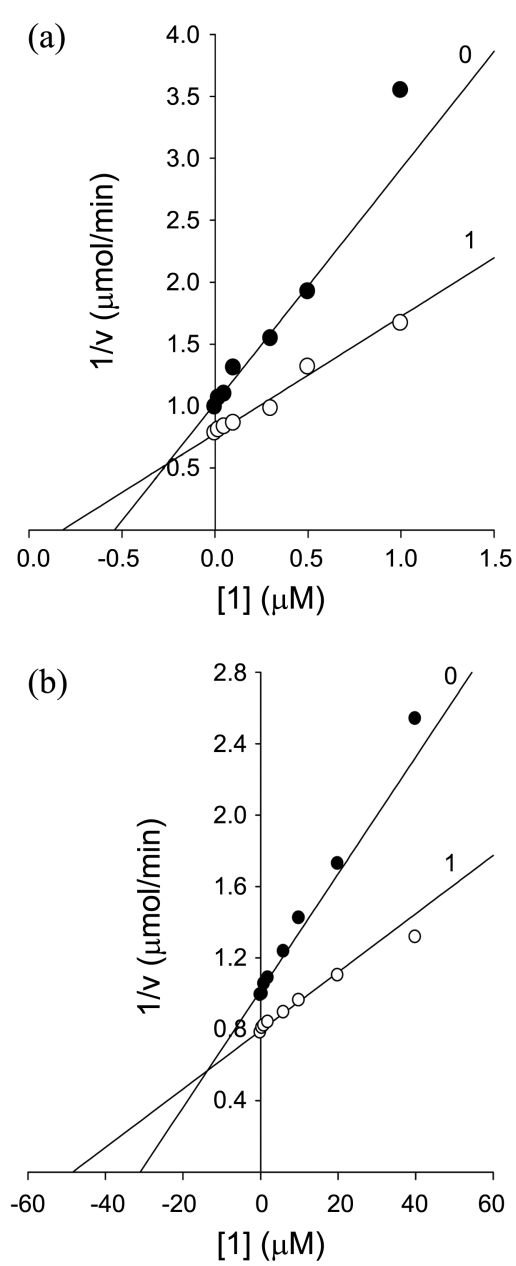

Figure 3. Dixon plots of 13-HPOD generation by soybean lipoxygenase- 1 in the presence of 7 (a) and 3 (b) at $25^{\circ} \mathrm{C}, \mathrm{pH} 8.0$. Concentrations of substrates for curves 0 and 1 were 20 and $40 \mu \mathrm{M}$, respectively. 


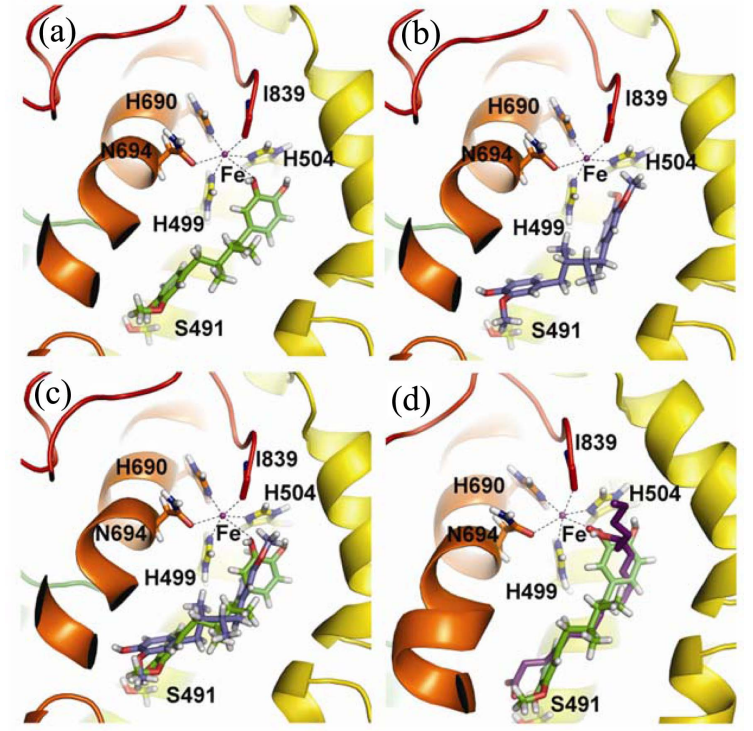

Figure 4. (a), (b). Binding orientation of 7 (green colored) \& 3 (blue colored) respectively at the substrate binding site of lipoxygenase-1. (c). Overlay of docked poses of 3 and 7. (d). Overlay of DDGA (7)-lipoxygenase-1 complex with the 13-S-HPOD (violetpurple colored)-lipoxygenase-3 complex (1IK3 pdb). All the figures were generated using PyMol version 0.97.

relationship among the dibenzylbutane lignans indicate that presence of steric groups such as methoxyl or 1,2-dioxomethylene bridge (compounds 1, 2, 4-6) at either ends of aromatic ring have resulted in loss of activity. Although the two active compounds $\mathbf{3}$ and $\mathbf{7}$ share structural similarity, they have a subtle variation in substitution - a hydroxyl to a methoxyl group at 3-position of ring A (Figure 1) contributing to significantly different inhibitory effect. Free 3-OH group seems to play a crucial role in the inhibition of lipoxygenase activity.

To better understand the underlying molecular mechanism of DDGA and MDGA-mediated inhibition on lipoxygenase, molecular docking studies involving were performed. The crystal structure of soybean lipoxygenase-1 features Fe metal atom ligated to conserved residues like three histidine residues - H499, H504, H690, asparagine 694 and the carboxylate moiety on isoleucine 839 . Even though soybean lipoxygensae is very similar to human lipoxygenase; it differs from human in having only three conserved histidine residues and asparagine 694 weakly ligated to $\mathrm{Fe}$, which are critical for enzymatic activity. The metalloenzyme shows an octahedral geometry by having coordination with three histidines, asparagine and isoleucine and the $6^{\text {th }}$ sphere is usually occupied by water molecule or the substrate-product or inhibitor. $^{15-18}$

Our molecular docking studies indicated that both DDGA (7) and MDGA (3) adopt a similar binding mode but the detailed interactions with metalloenzyme are different. DDGA (7), with its free phenolic hydroxyl group is able to ligate to Fe atom and forms the $6^{\text {th }}$ co-ordination sphere (Figure 4(a)), whereas MDGA (3), with its 3-methoxy group tilts away from the Fe as shown in Figures 4(b) and 4(c). Apart from ligation to $\mathrm{Fe}$ atom, all other van der Waals contacts with His504, Trp500, Leu546, Phe557, and Ser491 were seen with both compounds. Variations in the activity levels of these two compounds could well be attributed to interaction with $\mathrm{Fe}$ metal. Co-ordination to $\mathrm{Fe}$ atom is also seen with the ligands in crystal structures of lipoxygenase- $3^{19}$ (Figure 4(d)); as well as with (-)-epigallocatechin gallate, ${ }^{20}$ wherein the keto group of ligand is coordinated to the Fe atom and consistent with competitive mode of inhibition.

Based upon our data, we speculate that the differences in the inhibitory activity of these compounds can be ascribed to free phenolic hydroxyl group at the 3-position. Presence of steric groups like methoxyl or 1,2-dioxomethylene bridge at 3 -position hinders the activity as they seem to lose the contacts with Fe metal atom.

In summary, we have assessed the soybean lipoxygenase1 inhibitory activities of seven kinds of lignans (1-7) from nutmeg. We showed that the two dibenzylbutane lignans, MDGA (3) and DDGA (7) exhibited potent lipoxygenase inhibitory activities. Our SAR and molecular docking studies on MDGA (3) and DDGA (7) strongly corroborated the experimental enzyme inhibition results. They should have potential for use as an antioxidant additive in food. However, its biological significance in living systems is still largely unknown. Thus, it is not clear whether ingested MDGA (3) and DDGA (7) are absorbed into the system through the intestinal tract and delivered to the places where lipoxygenase inhibitors are needed. The relevance of the in vitro experiments in simplified systems to in vivo protection from oxidative damage should be carefully considered. Their further evaluation is needed to identify formulate a holistic and dynamic perspective.

\section{Experimental}

General. Soybean lipoxygenase-1 (EC 1.13.11.12, Type 1), linoleic acid and Tween-20 were purchased from Sigma (Sigma Chemical Co, St. Louis, MO, USA). Compounds 1-7 were obtained from our previous work. ${ }^{7}$ NDGA (nordihydroguaiaretic acid) was purchased from Aldrich (Milwaukee, WI, USA). All reagent grade chemicals were purchased from Sigma (St. Louis, MO, USA).

Enzyme Assay. The enzyme assay was performed as previously reported ${ }^{21}$ with a slight modification. Briefly, 10 $\mu \mathrm{L}$ of an ethanolic inhibitor solution was mixed with $60 \mu \mathrm{L}$ of $1 \mathrm{mM}$ stock solution of linoleic acid and $2.925 \mathrm{~mL}$ of 0.1 $\mathrm{M}$ Tris- $\mathrm{HCl}$ buffer (pH 8.0) in a quartz cuvette. Then, $5 \mu \mathrm{L}$ of a $0.1 \mathrm{M}$ Tris-HCl buffer solution ( $\mathrm{pH}$ 8.0) of lipoxygenase $(1.02 \mu \mathrm{M})$ was added. The resultant solution was mixed well followed by reading at $234 \mathrm{~nm}$ for $5 \mathrm{~min}$, which represents the formation of conjugated diene hydroperoxide (13HPOD, $\varepsilon=25000 \mathrm{M}^{-1} \mathrm{~cm}^{-1}$ ). A lag-period shown in the lipoxygenase reaction ${ }^{22}$ was excluded for the determination of initial rates. The stock solution of linoleic acid was prepared with methanol and Tris- $\mathrm{HCl}$ buffer at $\mathrm{pH} 8.0$, then total methanol content in the final assay was adjusted below $1.5 \%$. Two concentrations (20 and $40 \mu \mathrm{M})$ of linoleic acid 
were selected for Dixon plots. The assay was conducted in triplicate of separate experiments. The data analysis was performed by using Sigma Plot 2000 (SPSS Inc., Chicago, IL). The inhibitory concentration leading to $50 \%$ activity loss $\left(\mathrm{IC}_{50}\right)$ was obtained by fitting experimental data to the logistic curve by the equation as follows:

$$
\text { Activity }(\%)=100\left[1 /\left(1+\left([\mathrm{I}] / \mathrm{IC}_{50}\right)\right)\right]
$$

Inhibition mode was analyzed by Enzyme Kinetics Module 1.0 (SPSS Inc.) equipped with Sigma Plot 2000.

Molecular Docking. All the simulations were performed on Linux workstations using InsightII 2005 software package (Accelrys, CA, USA). The crystal structure of soybean lipoxygenase-1 (PDB ID: $1 \mathrm{YGE}$ ) determined at $1.4 \AA$ resolution $^{23}$ was used for the molecular docking studies. Hydrogens, charges and potentials were assigned using the CVFF force field and the protein was energy minimized. For the ligand preparation, DDGA (7) and MDGA (3) were sketched with the help of Builder module in InsightII 2005. Then the charges \& potentials were assigned using CVFF forcefield and the minimization was done using the Discover module in InsightII 2005 with 1000 steps of steepest descents followed by 5000 steps of conjugate gradients. For molecular docking, the program GOLD (Genetic Optimisation for Ligand Docking, Cambridge Crystallographic Data Centre, $\mathrm{UK})^{24}$ was employed to dock the DDGA (7) and MDGA (3) into the substrate binding site of soybean lipoxygenase. Active site radius of $10.0 \AA$ was defined from the Fe metal atom coordinated to H499, H504, H690, N694, I839 residues. The RMS deviation was considered within $1.5 \AA$ and the annealing parameter of van der vaals interaction was 4.0 Á, hydrogen bond interaction was 2.5 Á.

Acknowledgments. This work was supported by grants from the Priority Research Centers Program (2012-0006683) of the Ministry of Education, Science and Technology, republic of Korea and by a grant from the Bio-Synergy Research Project (NRF-2013M3A9C4078145) of the Ministry of Science, ICT and Future Planning through the National Research Foundation.

\section{References}

1. Calliste, C. A.; Kozlowski, D.; Duroux, J. L.; Champavier, Y.; Chulia, A. J.; Trouillas, P. Food chem. 2010, 118, 489.

2. Chung, J. Y.; Choo, J. H.; Lee, M. H.; Hwang, J. K. Phytomedicine 2006, 13, 261.

3. Jin, D.-Q.; Lim, C. S.; Hwang, J. K.; Ha, I.; Han, J.-S. Biochem. Biophys. Res. Commun. 2005, 331, 1264.

4. Yang, S.; Na, M. K.; Jang, J. P.; Kim, K. A.; Kim, B. Y.; Sung, N. J.; Oh, W. K.; Ahn, J. S. Phytother. Res. 2006, 20, 680.

5. Morita, T.; Jinno, K.; Kawagishi, H.; Arimoto, Y.; Suganuma, H.; Inakuma, T.; Sugiyama, K. J. Agric. Food Chem. 2003, 51, 1560.

6. Muchtaridi; Anas Subarnas, A.; Apriyantono, A.; Mustarichie, R. Int. J. Mol. Sci. 2010, 11, 4771.

7. Kwon, H. S.; Kim, M.-J.; Jeong, H. J.; Yang, M. S.; Park, K. H.; Jeong, T.-S.; Lee, W. S. Bioorg. Med. Chem. Lett. 2008, 18, 194.

8. Maeda, A.; Tanimoto, S.; Abe, T.; Kazama, S.; Tanizawa, H.; Nomura, M. Yakugaku Zasshi. 2008, 128, 129.

9. Cornicelli, J. A.; Trivedi, B. K. Curr. Pharm. Des. 1999, $5,11$.

10. Kris-Etherton, P. M.; Keen, C. L. Curr. Opin. Lipidol. 2002, 13, 41.

11. Grechkin, A. Prog. Lipid Res. 1998, 37, 317.

12. Richard-Forget, F.; Gauillard, F.; Hugues, M.; Jean-Marc, T.; Boivin, P.; Nicolas, J. J. Food Sci. 1995, 60, 1325.

13. Berry, H.; Debat, H.; Larreta-Garde, V. FEBS Lett 1997, 408, 324.

14. Schilstra, M. J.; Veldink, G. A.; Verhagen, J.; Vliegenthart, J. F. Biochemistry 1992, 31, 7692.

15. Minor, W.; Steczko, J.; Stec, B.; Otwinowski, Z.; Bolin, J. T.; Walter, R.; Axelrod, B. Biochemistry 1996, 35, 10687.

16. Skrzypczak-Jankun, E.; Amzel, L. M.; Kroa, B. A.; Funk, M. O., Jr. Proteins 1997, 29, 15.

17. Tomchick, D. R.; Phan, P.; Cymborowski, M.; Minor, W.; Holman, T. R. Biochemistry 2001, 40, 7509.

18. Andreou, A.; Feussner, I. Phytochemistry 2009, 70, 1504.

19. Skrzypczak-Jankun, E.; Bross, R. A.; Carroll, R. T.; Dunham, W. R.; Funk, M. O., Jr. J. Am. Chem. Soc. 2001, 123, 10814.

20. Skrzypczak-Jankun, E.; Zhou, K.; Jankun, J. Int. J. Mol. Med. 2003, 12, 415.

21. Rickert, K. W.; Klinman, J. P. Biochemistry 1999, 38, 12218.

22. Ruddat, V. C.; Whitman, S.; Holman, T. R.; Bernasconi, C. F. Biochemistry 2003, 42, 4172.

23. Minor, W.; Steczko, J.; Stec, B.; Otwinowski, Z.; Bolin, J. T.; Walter, R.; Axelrod, B. Biochemistry 1996, 35, 10687.

24. Jones, G.; Willett, P.; Glen, R. C.; Leach, A. R.; Taylor, R. J. Mol. Biol. 1997, 267, 727. 